\title{
A HAIR AND HAIR VORTEX SIMULATION TECHNIQUE BASED ON VECTOR FIELDS ON A MANIFOLD
}

\author{
Nguyen Van Huan \\ Information and Communication Technology University - Thai Nguyen University, Quyet Thang \\ Commune, Thai Nguyen City, Thai Nguyen Province.
}

Email: nvhuan@ictu.edu.vn

Received: 2 April 2015; Accepted for publication: 20 November 2015

\begin{abstract}
Hair is an important component in the virtual human simulation system. During hair simulation, not only the time required to ensure that there are required to express the physical characteristics, chemical such as hair styles, colors, curves, twists, parting one's hair and hair vortex (swirl), ... to represent the authenticity of the hair and improve hair simulation quality. While the studies has announced today that they mainly focus on simulating the styles, the motion of the hair that has not been expressed characteristics of hair as hair vortex,...

The paper introduces the concept of scalp model as a manifold in 3-dimensional space. Based on the nature of the singularity of the vector field on the manifold, the paper proposes a hair simulation technique on the scalp surface model based on vector field on the manifold. Thus, we can simulate appropriately the hair vortex on the scalp model.
\end{abstract}

Keywords: scalp, manifold, vector field, singularity, hair.

\section{INTRODUCTION}

In the system of virtual human simulation, hair is an important component and integral, contributing to the authenticity system. However, hair is a complex object with numbers in the hundreds of thousands of fibers per person, with many different styles, colors, curves, twists $[1,2]$. Besides, hair has localism with different physical and chemical features as hair swirl,... So, in hair simulation, one task is always set simulation requirements ensuring reduced time required but must meet quality requirements, expressing the detailed characteristics of the hair.

Currently, a hair simulation problem has received much attention of many research scientists in Vietnam and the world [1, 2, 3]. Depending on different applications, hair simulation problems are classified according to two main approaches to simulation blocks and strands form.

Two above approaches, usually the hair simulation as strands is used many for those applications that require about high real of hair results. Because hair simulation as strands allows simulating the interactions of hair, the local hair place, convex, concave, curvature, especially expressing hairstyles, including hair vortex characteristics, parted hair. 
In 2000, Hadap and Magnenat-Thalmann [4] based on the flow layers, swirl wind and light source around the block to capture hairstyles and simulate hair shapes. In 2002, Anjyo, Usami and T. Kurihara [5] have simulated hairstyles based on multiple light beams to obtain the strand of hair styles. In 2004, Noble[6], Kim[7] and Xu[8] was based on NURBS to represent hair, interaction and simulation of hair motion. In 2007, Hong Chen and Zhu [3] have simulated hairstyles by sketching the major hair curves based on vector field to guide the strands of hair, means that the authors have done sketch curves, the hairstyles from real hair photos (Figure 1a), means the vector field generated from set of sample vectors that distinguish different hair positions (Figure 1b), finally, simulating hairstyles from the vector field (Figure 1c).
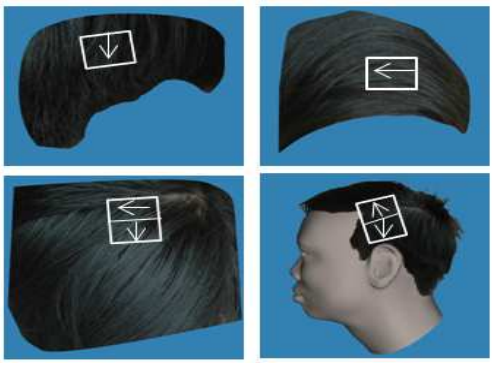

(a)

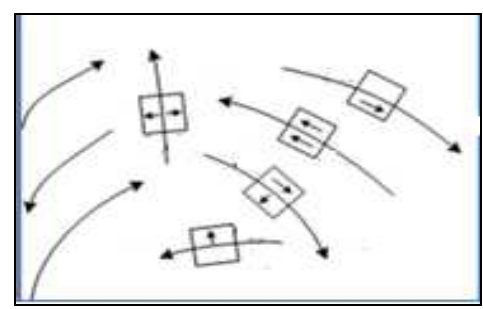

(b)

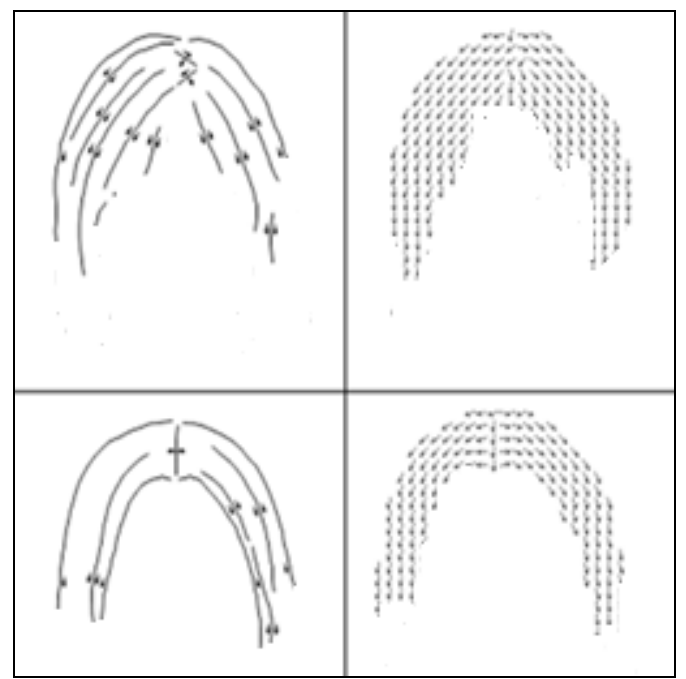

(c)

Figure 1. Simulating hair based on vector field.

However, above research works concentrate just on simulating hairstyles, hair motion on the basis of hair orientation based on vector field that is not performing all the characteristics of hair as hair swirl,...

On the basis of the results of research works $[9,10]$, the paper introduces the concept of scalp model as a manifold in 3-dimensional space. Based on the nature of the singularity of the vector field on the manifold, the paper proposes a hair simulation technique on the scalp surface model based on vector field on the manifold. Thus, we can simulate appropriately the hair swirl on the scalp model.

The remainder of the paper is shown as follows: Section 2 presents some basic concepts of manifolds, scalp surface model, vector fields, vector fields on the scalp manifold. Section 3 proposes a hair express algorithm based on vector fields on the scalp manifold. Next is testing and finally, conclusions and technical proposals. 


\section{SCALP MANIFOLD}

\subsection{A number of related concepts}

\section{Definition 1 (Topological manifold) [11,12]}

For $\mathrm{M}$ is a Hausdorff topo space $\mathrm{R}^{\mathrm{n}}$. A chart on $\mathrm{M}$ is a pair $(\mathrm{V}, \varphi)$, with $\mathrm{V}$ is an open set of $\mathrm{M}, \varphi: \mathrm{V} \rightarrow \mathrm{V}^{\prime}$ is a homeomorphism from $\mathrm{V}$ to a set of open $\mathrm{V}^{\prime}$ of $\mathrm{R}^{\mathrm{n}}$.

If $(\mathrm{V}, \varphi)$ is a chart on $\mathrm{M}$ then with all $\mathrm{x} \in \mathrm{V}, \varphi(\mathrm{x}) \in \mathrm{V}^{\prime}, \varphi(\mathrm{x})=\left(\mathrm{x}^{1}, \mathrm{x}^{2}, \ldots, \mathrm{x}^{\mathrm{n}}\right) \in \mathrm{R}^{\mathrm{n}}$, and then, the number of $x^{i}, i=1, \ldots, n$ is called the local coordinates of $x$.

If there is a family of maps $\left\{\left(\mathrm{V}_{\mathrm{i}}, \varphi_{\mathrm{i}}\right)\right\}_{\mathrm{i} \in \mathrm{I}}$ of $\mathrm{M}$ where $\left(\mathrm{V}_{\mathrm{i}}\right)_{\mathrm{i} \in \mathrm{I}}$ is an open cover of $\mathrm{M}$ then $\left\{\left(\mathrm{V}_{\mathrm{i}}, \varphi_{\mathrm{i}}\right)\right\}_{\mathrm{i} \in \mathrm{I}}$ is called a atlat (set of charts) of $\mathrm{M}$. Topological space $\mathrm{M}$ has an atlas is called topological k-manifold, where $\mathrm{k}$ is the number of variables used in expressing charts.

\section{Definition 2 (Differentiable Atlat) [11,12]}

For $\mathrm{M}$ is a topological k-manifold in space $\mathrm{R}^{\mathrm{n}}$. Atlat $\left\{\left(\mathrm{V}_{\mathrm{i}}, \varphi_{\mathrm{i}}\right)\right\}_{\mathrm{i} \in \mathrm{I}}$ is a differentiable atlat of $\mathrm{M}$, If two arbitrary charts of atlat $\left(\mathrm{V}_{1}, \varphi_{1}\right),\left(\mathrm{V}_{2}, \varphi_{2}\right)$ that $\mathrm{V}_{1} \cap \mathrm{V}_{2} \neq \varnothing, \varphi_{1}: \mathrm{V}_{1} \rightarrow \mathrm{V}^{\prime}{ }_{1}, \varphi_{2}: \mathrm{V}_{2} \rightarrow \mathrm{V}^{\prime}{ }_{2}$, then mapped:

$\left.\varphi_{2} \cdot \varphi_{1}^{-1}\right|_{\varphi 1(\mathrm{~V} 1 \cap \mathrm{V} 2)}: \varphi_{1}\left(\mathrm{~V}_{1} \cap \mathrm{V}_{2}\right) \rightarrow \varphi_{2}\left(\mathrm{~V}_{1} \cap \mathrm{V}_{2}\right)$ is a differentiable mapping.

Figure 2 below illustrates about differentiable Atlas of $\mathrm{M}$.

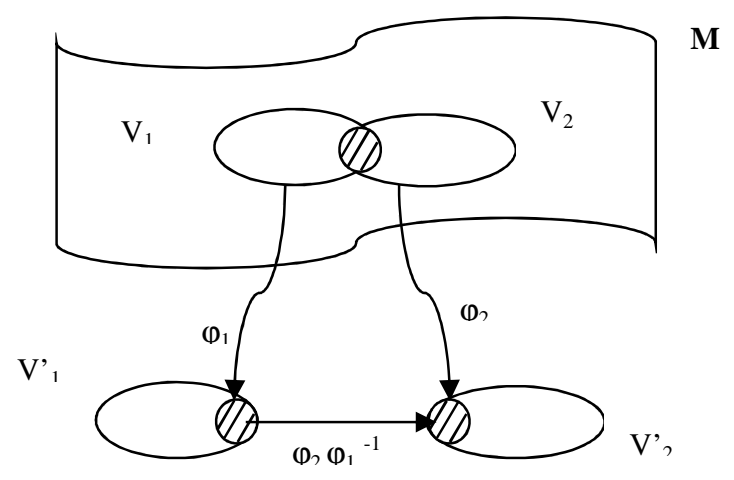

Figure 2. Differentiable Atlas of M.

Definition 3 (Differentiable Manifold) [11, 12]

For $\mathrm{M}$ is a topological k-manifold in space $\mathrm{R}^{\mathrm{n}}$, with $\left\{\left(\mathrm{V}_{\mathrm{i}}, \varphi_{\mathrm{i}}\right)\right\}_{\mathrm{i} \in \mathrm{I}}$ and $\left\{\left(\mathrm{V}_{\mathrm{j}}, \psi_{\mathrm{j}}\right)\right\}_{\mathrm{j} \in \mathrm{J}}$ are differentiable atlats of $M$. Then, $M$ is called a differentiable $k$-manifold, If $\left\{\left(V_{i}, \varphi_{i}\right),\left(V_{j}, \psi_{j}\right)\right\}_{i \in I, j \in J}$ is also a differentiable atlat.

Definition 4 (Vector Field) $[11,12]$ 
For $\mathrm{M}$ is a differentiable k-manifold in space $\mathrm{R}^{\mathrm{n}}$. Then, $\mathrm{X}$ is called a vector field on a manifold $M$, If $X$ is a mapping from $M$ to $R^{n}$ and changing each point $p \in M$ into vector $X(p) \in T_{p} M$, where $T_{p} M$ is tangent space of $X(p)$ at point $p$ on $M$. Figure 2 below illustrates about Vector field $\mathrm{X}(\mathrm{p})$ on manifold $\mathrm{M}$.

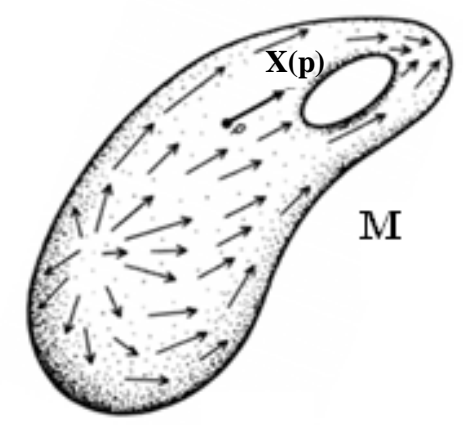

Figure 3. Vector field $X(p)$ on manifold $M$.

\subsection{Scalp surface model}

\section{Definition 5 (Scalp surface model)}

Scalp surface model $\mathrm{C}$ in space $\mathrm{R}^{3}$ is defined by the following formula:

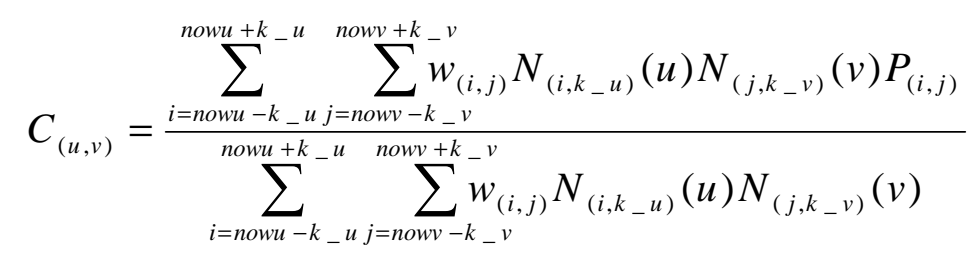

where: + Control points are $(\mathrm{n}+1)(\mathrm{m}+1)$.

+ nowu, nowv is the value of the order of considered points at which the two variables are $\mathrm{u}, \mathrm{v}$.

$+\mathrm{P}_{(\mathrm{i}, \mathrm{j})}$ are control points, $\mathrm{w}_{(\mathrm{i}, \mathrm{j})}$ is the weight corresponding to each $\mathrm{P}_{(\mathrm{i}, \mathrm{j})}$.

$+\mathrm{k} \_\mathrm{u}, \mathrm{k} \_\mathrm{v}$ is the degree of the basis functions in the two regions $\mathrm{u}, \mathrm{v}$ respectively.

$+\mathrm{N}_{\left(\mathrm{i}, \mathrm{k} \_\mathrm{u}\right)}(\mathrm{u}), \mathrm{N}_{\left(\mathrm{j}, \mathrm{k} \_\mathrm{v}\right)}(\mathrm{v})$ is the B-Spline basis functions corresponding to the two regions $\mathrm{u}, \mathrm{v}$.

Because two basis functions $\mathrm{N}_{\left(\mathrm{i}, \mathrm{k}_{-} \mathrm{u}\right)}(\mathrm{u})$ and $\mathrm{N}_{\left(\mathrm{j}, \mathrm{k}_{-} \mathrm{v}\right)}(\mathrm{v})$ are represented by two identical formulas, differing only in the indexes $i, j$ and degrees $k_{-} u, k_{-} v$. So, this section only presents equation describing the basis function $\mathrm{N}_{\left(\mathrm{i}, \mathrm{k}_{-} \mathrm{u}\right)}(\mathrm{u})$ corresponding to the index $\mathrm{i}$ and degree $\mathrm{k} \_\mathrm{u}$, remaining equation describing the basis function $\mathrm{N}_{\left(\mathrm{j}, \mathrm{k}_{-} \mathrm{v}\right)}(\mathrm{v})$ corresponding to the index $\mathrm{j}$ and degree $\mathrm{k} \_\mathrm{v}$ is presented similar to the basis function $\mathrm{N}_{\left(\mathrm{i}, \mathrm{k} \_\mathrm{u}\right)}(\mathrm{u})$.

* Formula describing the basis function $\mathrm{N}_{\left(\mathrm{i}, \mathrm{k} \_\mathrm{u}\right)}(\mathrm{u})$ corresponding to the index $\mathrm{i}$ and degree $\mathrm{k} \_\mathrm{u}$ :

$$
\begin{aligned}
& N_{(i, 1)}(u)=\left\{\begin{array}{l}
1: \text { knot }_{i} \leq u<\text { knot }_{i+1} \\
0: u \notin\left[\text { knot }_{i}, \text { knot }_{i+1}\right.
\end{array}\right) \\
& N_{\left(i, k_{-} u\right.}(u)=\frac{u-\text { knot }_{i}}{\text { knot }_{i+k_{-} u-1}-\text { knot }_{i}} N_{\left(i, k_{-} u-1\right)}(u)+\frac{\text { knot }_{i+k_{-} u}-u}{\text { knot }_{i+k_{-} u}-\text { knot }_{i+1}} N_{\left(i+1, k_{-} u-1\right)}(u)
\end{aligned}
$$


where: $+\mathrm{k} \_\mathrm{u}$ is degree of the B-Spline basis functions to the variable $\mathrm{u}$.

$+\mathrm{knot}_{\mathrm{i}}$ are knot vectors, is determined by the following formula:

$$
\text { knot }_{i}=\left\{\begin{array}{cc}
0 & 0 \leq i<k_{-} u \\
\left(i-k_{-} u+1\right) /\left(n-k_{-} u+1\right) & k_{-} u \leq i<n \\
1 & n \leq i<n+k_{-} u
\end{array}\right.
$$

* Formula describing the basis function $\mathrm{N}_{(\mathrm{j}, \mathrm{k}-\mathrm{v})}(\mathrm{v})$ corresponding to the index $\mathrm{j}$ and degree $\mathrm{k}_{-} \mathrm{v}$ :

$$
\begin{aligned}
& N_{(j, 1)}(v)=\left\{\begin{array}{l}
1: \text { knot }_{j} \leq v<\text { knot }_{j+1} \\
0: \mathrm{v} \notin\left[\text { knot }_{j}, \text { knot }_{j+1}\right)
\end{array}\right. \\
& N_{\left(j, k_{-} v\right)}(v)=\frac{v-\text { knot }_{j}}{\text { knot }_{j+k_{-} v-1}-\text { knot }_{j}} N_{\left(j, k_{-} v-1\right)}(v)+\frac{\text { knot }_{j_{+k_{-} v}}-v}{\text { knot }_{j_{+k_{-} v}-\text { knot }_{j+1}}} N_{\left(j+1, k_{-} v-1\right)}(v)
\end{aligned}
$$

Where: + $\mathrm{k}_{-} \mathrm{v}$ is degree of the B-Spline basis functions to the variable $\mathrm{v}$.

$+\mathrm{knot}_{\mathrm{j}}$ are knot vectors, is determined by the following formula:

$$
\text { knot }_{j}=\left\{\begin{array}{cc}
0 & 0 \leq j<k_{-} v \\
\left(j-k_{-} v+1\right) /\left(n-k_{-} v+1\right) & k_{-} v \leq j<n \\
1 & n \leq j<n+k_{-} v
\end{array}\right.
$$

Figure 4 is scalp surface model $\mathrm{C}$, with each point $\mathrm{C}_{(\mathrm{u}, \mathrm{v})}$ in surface $(\mathrm{u}, \mathrm{v})$, then there will be corresponding point $\mathrm{p}^{\prime}(\mathrm{x}, \mathrm{y}, \mathrm{z})$ on $\mathrm{C}$ in $3 \mathrm{D}$ space.

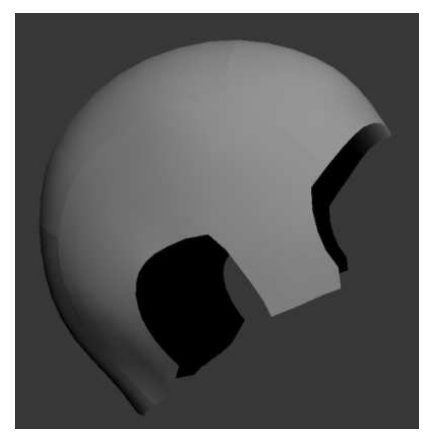

Figure 4. Scalp surface model C.

Corollary 1: The scalp surface model $C$ is a differentiable 2-manifold in space $R^{3}$

Proof: Indeed, based on the representation formula $\mathrm{C}$ in definition 5, function $\mathrm{C}_{(\mathrm{u}, \mathrm{v})}$ is expressed on the sum of B-Splines. So, $\mathrm{C}_{(\mathrm{u}, \mathrm{v})}$ is a differentiable function with two variables $\mathrm{u}, \mathrm{v}$.

Moreover, function $\mathrm{C}_{(\mathrm{u}, \mathrm{v})}$ is a differentiable mapping because it is expressed on B-Spline. So, square mapping $(\mathrm{u}, \mathrm{v})$ with $0<\mathrm{u}<1,0<\mathrm{v}<1$ from 2D model to 3D scalp surface model is mapped $1-1$. Thus, $\mathrm{C}_{(\mathrm{u}, \mathrm{v})}$ is a differentiable manifold, therefore, its image is also a differentiable manifold. Inferred that must prove: The scalp surface model is defined by $\mathrm{C}_{(\mathrm{u}, \mathrm{v})}$ with two variables $\mathrm{u}, \mathrm{v}$ is a differentiable 2-manifold in space $\mathrm{R}^{3}$. 


\section{HAIR SIMULATION ALGORITHM BASED ON VECTOR FIELD ON THE MANIFOLD}

In hair simulation, one of the indispensable tasks which are the modeling of the scalp surface model, because the scalp surface where hair grows. So, modeling and control scalp surface model has a key role to support the hair simulation and improve simulation efficiency, expressing hairstyles and detailed characteristics of hair such as hair swirl,...

In this section, this article will consider the hair performance on the vector field on the scalp manifold based on the idea of the Poincare-Hopf $[13,14,15,16]$ theorem, and considering the surface of the scalp as a differentiable 2-manifold in 3D space and studying characteristics on the surface of the scalp, hair and hair swirling on the surface of the scalp along with studying the characteristics of the theory of manifold, vector fields on manifolds to simulate hair swirling, showing the correspondence between the actual and simulation models in terms of mathematical.

\section{Several declarations and conventions}

- Building exposure space $\alpha_{\mathrm{i}}$, where $\alpha_{\mathrm{i}}$ is built based on the mean vector of the exposure vectors $\overrightarrow{T X}_{j}$ at each point $P_{i} \in C$ (scalp surface). In particular, the identification $\overrightarrow{T X}_{j}$ at each point $\mathrm{P}_{\mathrm{i}}$ can occur following three cases:

+ If points $P_{i}$ are the vertexes of the scalp surface mesh $C$, then $\overrightarrow{T X}_{j}$ are the exposure vectors of triangles that share a vertex $P_{i}$. Then, each $\vec{u}_{i}$ is the average of $\overrightarrow{T X}_{j}$ at each point $P_{i}$ (Figure 5a).

+ If points $\mathrm{P}_{\mathrm{i}}$ in each triangle, then each $\overrightarrow{T X}_{\mathrm{j}}$ is the exposure vectors of each that triangle. Then, each $\overrightarrow{\mathrm{u}}_{\mathrm{i}}$ is a $\overrightarrow{\mathrm{TX}}_{\mathrm{j}}$ and $\alpha_{\mathrm{i}}$ are triangles (Figure $5 \mathrm{~b}$ ).

+ If points $P_{i}$ belong to the edge of the triangles, then $\overrightarrow{T X}_{j}$ are the two exposure vectors of two triangles that share a edge at point $P_{i}$. Then, each $\vec{u}_{i}$ is the average of two $\overrightarrow{T X}_{j}$ at each $P_{i}$ (Figure 5c).

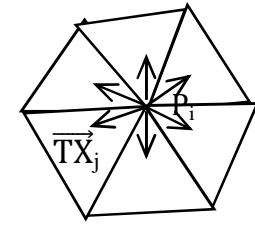

a)

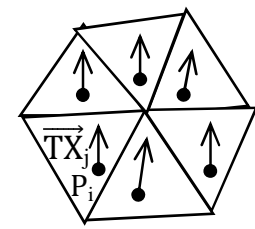

b)

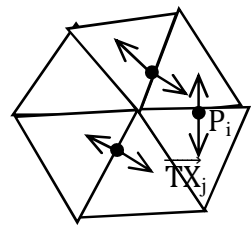

c)

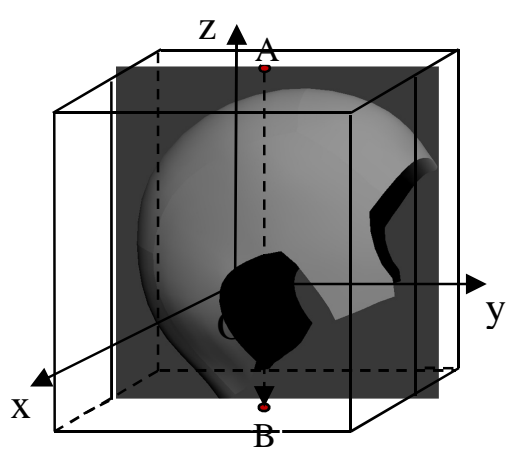

d)

Figure 5. The exposure vector $\overrightarrow{\boldsymbol{T}}_{j}$, axial coordinates $O z$ and $C$.

- Origin $\mathrm{O}$ coincides with the center of the scalp surface $\mathrm{C}$ and axial coordinates $\mathrm{Oz}$ coincides with the direction of the vector direction $\overrightarrow{A B}$ of $C$. In Figure 5d) including: points A, B respectively the center of the top and bottom of the $\mathrm{C}, \overrightarrow{\mathrm{AB}}$ is considered vector direction of the scalp surface $\mathrm{C}$. 
- $\vec{v}_{i}$ are the unit vectors, length is 1 and the first point is $P_{i}$, direction $\vec{v}_{i}$ same direction as the vectors $\overrightarrow{\mathrm{OP}}_{\mathrm{i}}$ (with $\mathrm{O}$ is the original coordinates).

- Vector field X consists of a set of $\vec{v}_{i}^{\prime}$ exposure vectors. Vectors $\vec{v}_{i}$ ' are the image of the vectors $\vec{v}_{i}$ with the first point $P_{i}$, The vectors $\vec{v}_{i}$ ' are built by performing parallel projection $T$ with z-axis to the vectors $\vec{v}_{i}$ on the exposure space plane $\alpha_{i}$.

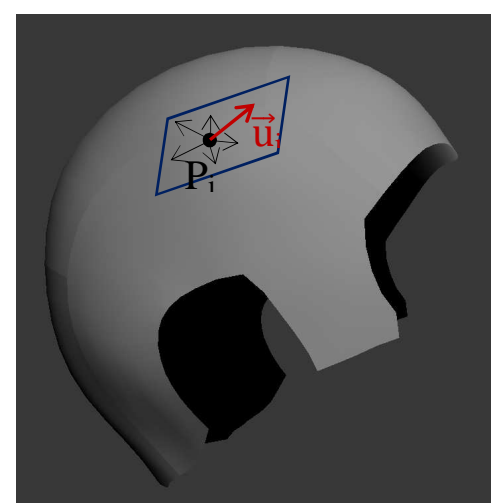

a) Building exposure space $\alpha_{i}$ with the average vector $\overrightarrow{\mathrm{u}}_{\mathrm{i}}$ of the exposure vectors $\overrightarrow{\mathrm{TX}}_{\mathrm{j}}$ at each point $\mathrm{P}_{\mathrm{i}}$

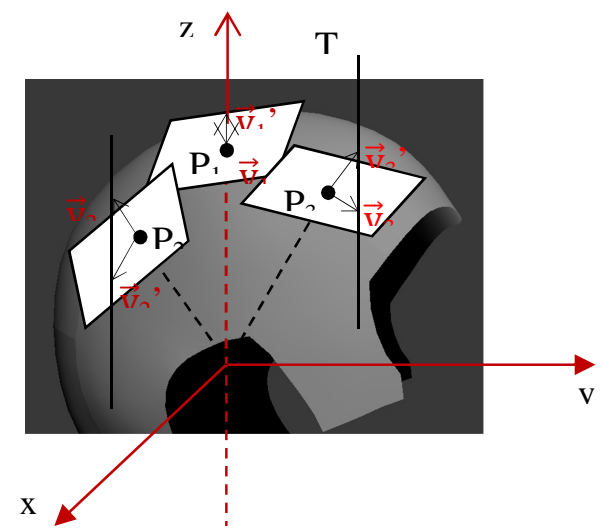

b) Building direction of the exposure vectors $\vec{v}_{\mathrm{i}}$ ' is the image of the vectors $\vec{v}_{i}$ with the first point $P_{i}$ based on $T$

Figure 6. Initializing the vector field $X$ that includes exposure vectors $\overrightarrow{\boldsymbol{v}}_{\boldsymbol{i}}^{\prime}$.

GHVF Algorithm (Generating Hairs based on Vector Field) executes hair simulation based on vector field (The GHVF algorithm is also presented in detail RAISE specification language [17]), includes specific steps:

The first step is initial the exposure space $\alpha_{i}$ and vector field $X$, including exposure vectors $\overrightarrow{\mathrm{v}}_{\mathrm{i}}{ }^{\prime}$ at each point $\mathrm{P}_{\mathrm{i}} \in \mathrm{C}$. Then, to calculate and check $\left|\overrightarrow{\mathrm{v}}_{\mathrm{i}}{ }^{\prime}\left(\mathrm{P}_{\mathrm{i}}\right)\right|$, If $\left|\overrightarrow{\mathrm{v}}_{\mathrm{i}}{ }^{\prime}\left(\mathrm{P}_{\mathrm{i}}\right)\right|=0$ then $\mathrm{P}_{\mathrm{i}}$ is a singularity point and putting wirl at point $\mathrm{P}_{\mathrm{i}}$. Otherwise, generating strands of hair $\mathrm{H}_{\mathrm{i}}$ at each point $\mathrm{P}_{\mathrm{i}} \in \mathrm{C}$ corresponding to each exposure vector $\vec{v}_{i}^{\prime}$. Finally, displaying strands of hair $\mathrm{H}_{\mathrm{i}}$ and XT on C.

\section{Hair simulation algorithm based on vector field GHVF}

Input: Scalp surface model $\mathrm{C}$ in space $\mathrm{R}^{3}$ is expressed by above definition $5, \mathrm{n}$ is the number of hairs (that want to initialize), Hair wirl XT.

Output: $\mathrm{n}$ the number of hairs $\mathrm{H}_{\mathrm{i}}$ and Hair wirl XT are initialized on $\mathrm{C}$.

\section{The detailed steps of the algorithm:}

\section{Begin}

Step 1: With $\forall \mathrm{P}_{\mathrm{i}} \in \mathrm{C}$

Building exposure space $\alpha_{i}$ by calculating the average exposure vector $\overrightarrow{\mathbf{u}}_{\mathrm{i}}$ of $\overrightarrow{\mathbf{T X}}_{\mathrm{j}}$.

$$
\overrightarrow{\mathrm{u}}_{\mathrm{i}}=\frac{\sum_{\mathrm{j}=1}^{\mathrm{m}} \overrightarrow{\mathrm{TX}}_{\mathrm{j}}}{\mathrm{m}}
$$

where $m$ is the number of exposure vectors $\overrightarrow{T X}_{j}$ at each point $P_{i} \in C$. 
Step 2: With $\forall \mathrm{P}_{\mathrm{i}} \in \alpha_{\mathrm{i}}$

Building the vector field $X$, including a set of vectors $\overrightarrow{\mathbf{v}}_{\mathrm{i}}{ }^{\prime}$ :

$$
X=\cup_{i=1}^{n} \overrightarrow{\mathbf{v}}_{\mathbf{i}}^{\prime}
$$

With the vectors $\overrightarrow{\mathbf{v}}_{\mathrm{i}}$ ' are defined by the projection $\mathrm{T}$ that parallel to the axis $\mathrm{z}$ to the vectors $\overrightarrow{\mathbf{v}}_{\mathrm{i}}$ on $\alpha_{\mathrm{i}}$ respectively ( $\overrightarrow{\mathbf{v}}_{\mathrm{i}}$, are the image of vectors $\overrightarrow{\mathbf{v}}_{\mathrm{i}}$ by T).

Step 3: If $\left|\overrightarrow{\mathbf{v}}_{\mathrm{i}}{ }^{\prime}\left(\mathrm{P}_{\mathrm{i}}\right)\right|=0$ then putting wirl $\mathrm{XT}$ at point $\mathrm{P}_{\mathrm{i}}\left(\mathrm{P}_{\mathrm{i}}\right.$ is a singularity point).

Otherwise, that is $\left|\overrightarrow{\mathbf{v}}_{\mathrm{i}}{ }^{\prime}\left(\mathrm{P}_{\mathrm{i}}\right)\right| \neq 0$ then generating strands of hair $\mathrm{H}_{\mathrm{i}}$ at each point $\mathrm{P}_{\mathrm{i}} \in \mathrm{C}$ corresponding to each exposure vector $\overrightarrow{\mathbf{v}}_{\mathrm{i}}$, of vector field $\mathrm{X}$ at points $\mathrm{P}_{\mathrm{i}}$.

Step 4: Displaying strands of hair $\mathrm{H}_{\mathrm{i}}$ and $\mathrm{XT}$ on $\mathrm{C}$.

End.

\section{Comments}

The hair simulation algorithm based on vector field GHVF ensure the soundness and the correct result.

Indeed, as the number of hairs on the scalp surface $\mathrm{C}$ of each person identified, so that the number of vertices $\mathrm{P}_{\mathrm{i}}$ on $\mathrm{C}$ is finite. This leads to the calculation of the average exposure vector $\overrightarrow{\mathbf{u}}_{\mathbf{i}}$ always done.

The algorithm uses the projection $\mathrm{T}$ parallel to the $\mathrm{z}$-axis, which means and vertical direction, making the projector will always exist at least one point $P_{i}$ where $\overrightarrow{\mathbf{v}}_{\mathrm{i}}{ }^{\prime}$ at $\left|\overrightarrow{\mathbf{v}}_{\mathrm{i}}{ }^{\prime}\left(\mathrm{P}_{\mathrm{i}}\right)\right|=0$. This is entirely consistent with actual per capita per person (usually at least a swirling hair).

Corollary 2: On the scalp surface model $C$ there exists at least one singular point $P_{i}$ at which $\left|\overrightarrow{\mathbf{v}}_{\mathrm{i}}{ }^{\prime}\left(\mathrm{P}_{\mathrm{i}}\right)\right|=0$

Proof: Because the coordinate origin is chosen to coincide with the center C, with the $\mathrm{z}$-axis is the vertical axis and the projection T for every vector $\overrightarrow{\mathbf{v}}_{\mathrm{i}}$ to $\alpha_{\mathrm{i}}$ exposure plane is parallel to the $\mathrm{z}$ axis (Figure 6).

On the other hand, the vectors $\overrightarrow{\mathbf{v}}_{i}$ have the same direction and with the direction of the vectors $\overrightarrow{\mathbf{O P}}_{\mathrm{i}}$, where $\mathrm{O}$ is the coordinate origin and $\mathrm{Oz}$ vertically so the vectors $\overrightarrow{\mathbf{O P}}_{\mathrm{i}}$ have the original $\mathrm{O}, \overrightarrow{\mathbf{v}}_{\mathrm{i}}$ rooted at $\mathrm{P}_{\mathrm{i}}$ will survive at least a direction vector which coincides with the direction of the $\mathrm{Oz}$ axis.

So, when making the projection T for every vector $\overrightarrow{\mathbf{v}}_{i}$ to contact plane $\alpha_{i}$ that parallel to the z-axis, then there exists at least one position $P_{i}$ at which the projection $\overrightarrow{\mathbf{v}}_{\mathbf{i}}$ ' of $\overrightarrow{\mathbf{v}}_{\mathbf{i}}$ was destructively, ie $\left|\overrightarrow{\mathbf{v}}_{\mathbf{i}}{ }^{\prime}\left(\mathbf{P}_{\mathbf{i}}\right)\right|=\mathbf{0}$.

\section{Detail specification on hair simulation algorithm GHVF}

The GHVF algorithm is specificated detail RAISE language (Rigorous Approach Industrial Software Engineering) [17]. The algorithm uses the basic style of RAISE specification language as: Nat-style as natural, Real-kind real number, Unit-type hollow, Bool - a logical, Point abstract style, Point-list style list.

The algorithm GHVF is specificated detail as follows:

scheme GHVF = 
class

type /* Scalp surface model $C$ with a set of vertices ps and surface */

$\mathrm{C}=\{\mid$ ps:Point-set:-faceconstraint(ps) $\mid\}$,

Hairstrands, XT,

/* Hairstyle, Hair Vortex XT */

Point $=$ Real $><$ Real $><$ Real,

C_Hair $=\mathrm{C}><$ Hairstrands $><\mathrm{XT}, \quad / *$ Scalp surface $C$ is coverred hair $*$

/* Create a UnitVector with lengths are equal to 1.0,

First point pi of $C$ and coincides with the direction vector $O P^{*} /$

UnitVector $=\{\mid(\mathrm{p}, \mathrm{q}):$ Point $>\langle$ Point:- distance $(\mathrm{p}, \mathrm{q})=1.0|\}$

value

faceconstraint:Point-set $\rightarrow$ Bool,

distance: Point $><$ Point $\rightarrow$ Real $\quad / *$ Two point distance $* /$

distance $((\mathrm{x} 1, \mathrm{y} 1, \mathrm{z} 1),(\mathrm{x} 2, \mathrm{y} 2, \mathrm{z} 2))$ is

$\operatorname{sqrt}((\mathrm{x} 1-\mathrm{x} 2) *(\mathrm{x} 1-\mathrm{x} 2)+(\mathrm{y} 1-\mathrm{y} 2) *(\mathrm{y} 1-\mathrm{y} 2)+(\mathrm{z} 1-\mathrm{z} 2) *(\mathrm{z} 1-\mathrm{z} 2))$,

sqrt: Real $\rightarrow$ Real,

*2 Square function */

thuoc:Point $><\mathrm{C} \rightarrow$ Bool, $\quad / *$ Function to determine the point on the surface $C *$ /

tiepxuc:UnitVector $><\mathrm{C} \rightarrow \mathbf{B o o l}, / *$ Function for exposed vector with surface $C * /$

$-:$ Point $><$ Point $\rightarrow$ Point,

/* Subtraction of two points */

constraint:C_Hair $\rightarrow$ Bool

constraint((headskinface, Hairstrands, XT)) is true,

VFCreator: $\mathrm{X} \rightarrow \mathrm{X}$,

/*Initialize vector field*/

vi: Point->UnitVector,

$+:$ Point $><$ UnitVector $\rightarrow$ Point, $/ *$ Plus a point with a vector $* /$

/* Construction of the exposure space oi based on the average vector $u$ of the

exposure vector at each point $\mathrm{Pi}$ \%/

Tinh_u:C $><$ Point $><$ UnitVector-list $><$ Nat- $>$ Point-list

Tinh_u(c, p,TX,n,m) as u_TB

post $\left(\right.$ all $\mathrm{i}, \mathrm{j}:$ Nat: $\left.-(\mathrm{i}<=\mathrm{n}) \wedge(\mathrm{j}<=\mathrm{m})=>\left(\mathrm{u}_{-} \mathrm{TB}(\mathrm{i})=\operatorname{sum}(\mathrm{TX}(\mathrm{j})) / \mathrm{m}\right)\right)$,

${ }^{*}$ Construction of exposure vector $v i$ ' is the vector image through projection of $T$ on the exposure space plane $\alpha i * /$

Tap_vi':C->UnitVector-set

Tap_vi'(T,vi,Pi) as vi’ post (all T, vi:UnitVector, Pi:Point:-true)

T_vi isin T_vi'=> thuoc $(\mathrm{p}, \mathrm{c}) \wedge \operatorname{tiepxuc}((\mathrm{p}, \mathrm{q}), \mathrm{c})$,

/* Initialize vector field $X$ on $C$ including UnitVectors with lengths is equal to 1.0, first point $p$ of $C$ and the direction of $X$ exposure to $C *$ /

$\mathrm{X}=\{|(\mathrm{c}, \mathrm{vs}): \mathrm{C}\rangle\langle$ UnitVector-set $:-$ if $\mathrm{vs}=\{\}$ then true else

all $(p, q)$ :vi':- $(p, q)$ isin vs=>thuoc $(p, c) \wedge \operatorname{tiepxuc}((p, q), c)$ 


\section{end $\mid\} / * X-$ Vector Field $* /$}

constraint: $\mathrm{X}><$ HairStrands $\rightarrow$ Bool,

$/ *$ Constraint function between the vector field $X$ and hair styling*/

HieuChinh: $\mathrm{X}><$ HairStrands $><\mathrm{XT} \rightarrow \mathrm{X}, /^{*}$ Correction function $X * /$

GHVF:C $><$ HairStrands $><\mathrm{XT} \rightarrow \mathrm{X}$

$\operatorname{GHVF}(\mathrm{c}$, hairstrands, $\mathrm{XT})$ is let $\mathrm{vf}=\mathrm{VFCreator}(\mathrm{c},\{\})$

in HieuChinh(vf, hairstrands,XT) end

axiom

all (c,vs),(c1, vs1):X, hairstrands:HairStrands:-

HieuChinh $((\mathrm{c}, \mathrm{vs})$, hairstrands, $\mathrm{XT})=(\mathrm{c} 1, \mathrm{vs} 1)=>$

(all $(p, q)$ : vi':- $(p, q)$ isin vs $\wedge$

if $\left(\mathrm{vi}^{\prime} \operatorname{isin}\{\}\right) \wedge((\mathrm{p}, \mathrm{q}) \sim \operatorname{isin} \mathrm{vs} 1) \wedge$

let $\mathrm{q} 1=\mathrm{p}+\mathrm{v}(\mathrm{p})$ in $(\mathrm{p}, \mathrm{q} 1)$ isin $\mathrm{vs} 1$ end

end

) ^constraint((c1, vs1),hairstrands,XT)

/* Show headskinface surface scalp is coverred hair and vortex*/

all (headskinface, hair,XT):C_Hair:- constraint((headskinface, HairStrands, XT))=true

end

\section{EXPERIMENTS}

GHVF Algorithm executes hair simulation on the scalp surface model of the Vietnamese and was installed experiment on PC computer with CPU T2050 @1.60 GHz, 1 GB RAM by Visual C++ software, OPENGL graphics library and with 10.000 strands of hair, width $=2.0$, length $=1.0$. Figure 7 is the result of hair models.

Figure 7 illustrates hairstyles given from the algorithm GHVF with 10,000 strands of hair initialized. Figure 7.a) shows hairstyles with a few different angles and the results show that the hairstyles are consistent with Vietnam.

In fact, the research work of the authors in Vietnam and the world, most of them do not focus on detailed feature simulation on hair as hair vortex, parted hair,...[3, 4, 5, 7, 8], such as the hair model on Figure 7.b) is hairstyle without vortex.

Simulating hairstyles with vortex as figure 7.c) contributes to further improvement the hair detailed features and support for hair simulation problems and virtual humans be more realistic.

So, we can say that the method proposed by the authors have differences and advantages than the methods of other authors [3, 7]: Simulations were swirling hair and identify the location of vortex on hair scalp surface automatically through the use of vector fields on manifolds. While the proposals of other authors [3, 7] without using vector fields on manifolds, so to simulate vortex or the characteristic hair, hair swirling and to determine the location hair swirling is very difficult and they often choose available one fixed position on the surface of the scalp to put hair swirling. In fact, we can not be so, because each person has a different hair swirling position. 

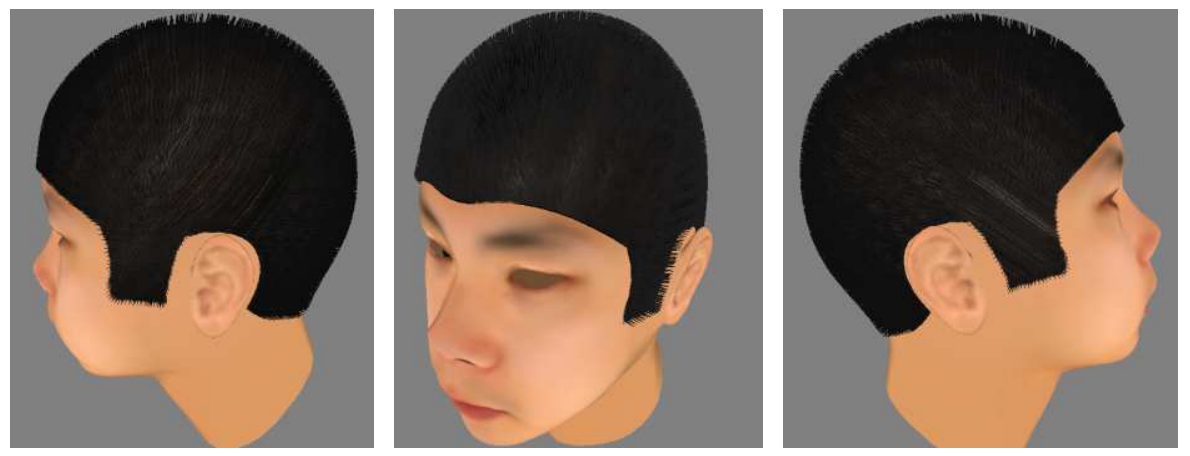

a) initial hair models

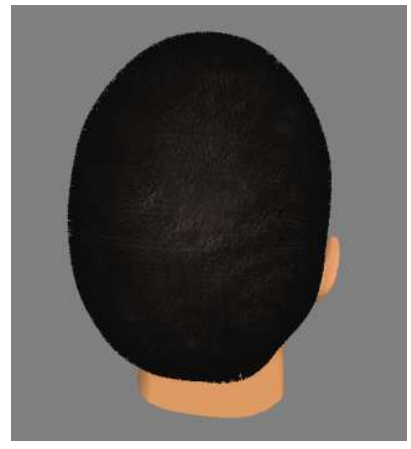

b) Hair model without wirl

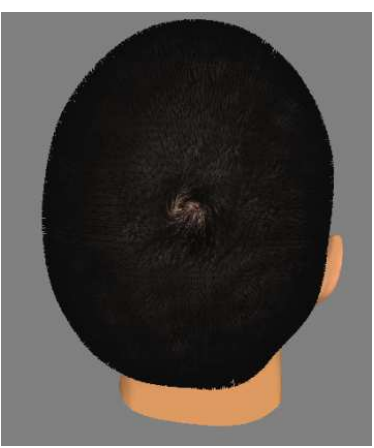

c) Hair model with wirl

Figure 7. hair models are simulated based on vector field on manifold.

To be able to see more clearly advantages of the proposed method in the author's article, the following article offers some comparisons and evaluations of image quality and timing simulation of the proposed method with suggestions of other works, in particular:

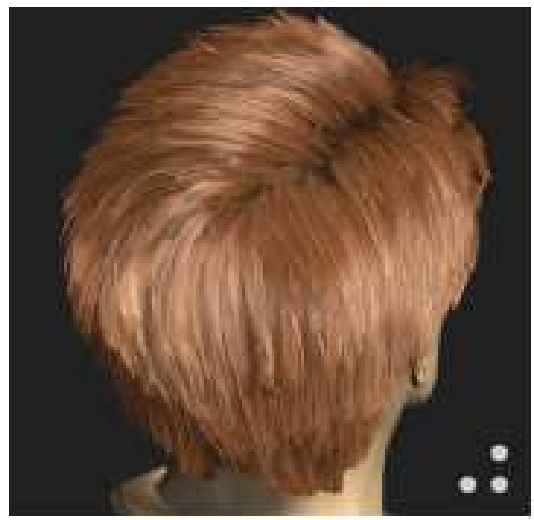

a) The model's hair style Chen and Zhu [3]

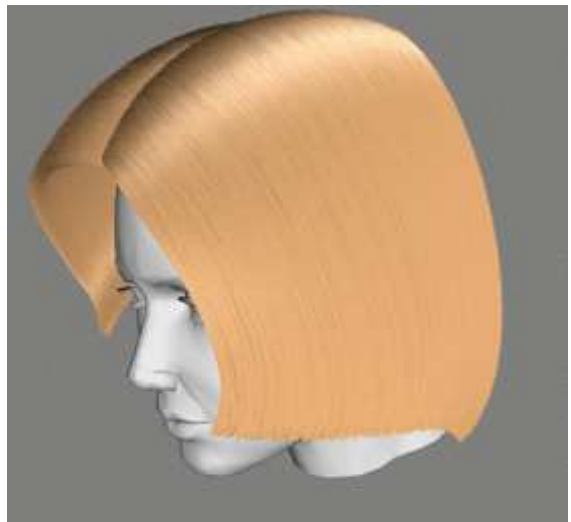

b) The model's hair style Kim and Neuman [7]

Figure 8. Some hairstyles. 
About simulation picture quality: The quality comparisons between the proposed method in the paper with the methods of other authors $[3,7]$ is very difficult, because each hair style of each person in each country is different from dependence on anthropology. So the hair can simulate different, especially those features as hair swirling. Furthermore, most of the buildings in the world have not focused or interested in simulating hair swirling, but the use of vector fields on manifolds in simulation even without. Therefore, the comparison of quality between the proposals in the paper with the other buildings in the world are not appropriate.

Look at the pictures on Figures $8 \mathrm{a}$ ) and $8 \mathrm{~b}$ ): The method of the authors [3,7] just stop and focus mainly on hair styling simulation, but not focus on hair swirling. Chen $\mathrm{H}$. and Zhu [3] Simulation hair styles by outlining the main hair curve based on vector field to guide gathers hair, ie the authors performed sketch the curves, the hair styles from actual hair photos (Figure 8a).

In terms of duration, speed of execution: The method proposed by the article would result in much faster than the methodology of other works $[3,7]$. The method proposed in the paper used for performing vector field, hair swirling simulation, specific points on the surface of the scalp. The surface of the scalp which is defined by NURBS modeling improved (Definition 5), while the methods of other works $[3,7]$ to use original NURBS model:

$$
C_{(u, v)}=\frac{\sum_{i=0}^{m} \sum_{j=0}^{n} w_{(i, j)} N_{\left(i, k_{-} u\right)}(u) N_{\left(j, k_{-} v\right)}(v) P_{(i, j)}}{\sum_{i=0}^{m} \sum_{j=0}^{n} w_{(i, j)} N_{\left(i, k_{-} u\right)}(u) N_{\left(j, k_{-} v\right)}(v)}
$$

The graph below proves the speed calculated between the method proposed in the paper using improved NURBS and original NURBS model.

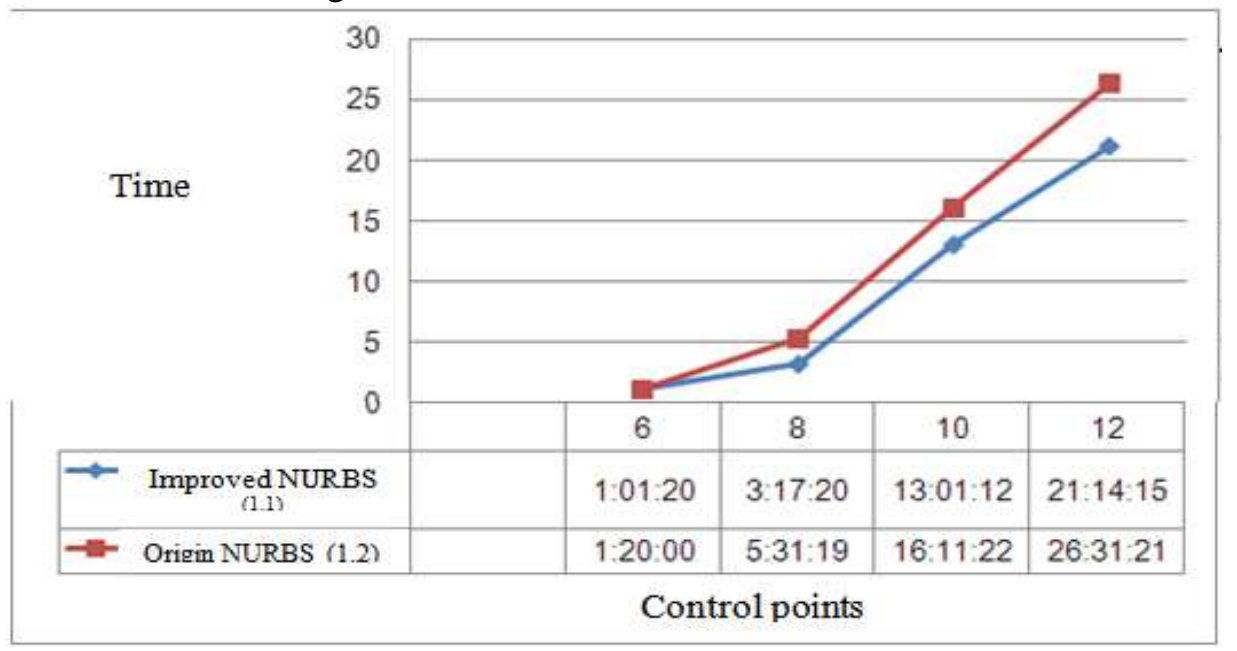

In above chart 1 , the comparison of the computation time when simulation hair, hair swirling using original NURBS (1.2) and NURBS improvement (1.1) corresponding to four different levels of the hair and control points: $6 ; 8 ; 10 ; 12$.

Thus, the complexity is compared as above and the chart 1 , showing hair simulation using NURBS modeling improvements in (1.1) have a huge advantage in terms of time than using the original NURBS model in (1.2). Due to the introduction of improved formula NURBS is essentially domain reduce the influence of the control points to a point under consideration, the only changes were local and not affect the entire object. 


\section{CONCLUSIONS}

Hair simulation is an important task in the virtual human simulation system, during hair simulation, not only the time required to ensure that there are also required to express the physical characteristics, chemical such as hair styles, colors, curves, twists, parting one's hair and hair swirl, ... and improving the quality of simulation.

This paper has proposed a hair simulation technique on the scalp surface model of the Vietnamese based on the vector field on the manifold and the basis of the concept of the scalp as a manifold in 3-dimensional space. Simultaneously, based on the nature of the singularity of the vector field on the manifold to be able to simulate the hair vortex properly.

Acknowledgements. The author would like to thank colleagues at VR Research - Institute of Information Technology under the Vietnam Academy of Science and Technology, who contributed valuable comments helped to author complete this paper quickly.

\section{REFERENCES}

1. Hadap S., Cani M. P., Lin M., Kim T. Y., Bertails F., Marschner S., Ward K., and Zoran K. - Strands and Hair Modeling, Animation and Rendering, Association for Computing Machinery's Special Interest Group on Computer Graphics and Interactive Techniques Course Notes, 2008.

2. Ward K., Bertails F., Kim T. Y., Arschner, Cani M. P., Lin M. - A Survey on Hair Modeling: Styling, Simulation and Rendering, University of North Carolina at Chapel Hill, 2007.

3. Chen H. and Zhu S. C. - A Generative Model of Human Hair for Hair Sketching, Department of Statistics, University of California, Los Angeles, 2007.

4. Hadap Z. and Magnenat-Thalmann N. - Interactive hair styler based on fluid flow, in Computer Animation and Simulation '00, Aug. 2000, pp. 87-100.

5. Anjyo K., Usami Y. and Kurihara T. - A simple method for extracting the natural beauty of hair, Proceedings of Association for Computing Machinery's Special Interest Group on Computer Graphics and Interactive Techniques, August 2002, pp. 111-120.

6. Noble P. and Tang W. - Modelling and animating cartoon hair with nurbs surfaces, in Computer Graphics International (CGI), June 2004, pp. 60-67.

7. Kim Y. and Neumann U. - A Thin Shell Volume for Modeling Human Hair, Computer Graphics and Immersive Technology Laboratory, Integrated Media Systems Center, University of Southern California, 2002.

8. Xu Z., Yang X. D., Wang T. and Yang J. - The cluster hair model, Graphics Models and Image Processing 62 (2) (2000) 85-103.

9. Huan N. V. and Binh P. V. - Representing the Hair Regions on the correlative Scalp Regions based on Vector Fields, Special issue of Research, Development and Application on Electronics, Journal of Telecommunications and Information Technology 24 (4) (2010) 5-14.

10. Huan N. V. and Toan D. N. - Vector Fields in Expressing Hairstyles, Proceedings of The 2nd IEEE International Conference on Advanced Computer Control -ICACC 2010, Shenyang 27-29/03/2010, China 2010, pp. 541-546.

11. Doanh N. V. - Differentiable Manifold, Publisher University of Pedagogical, 2007. 
12. Duong H. H. -Mathematical Analysis on Manifolds - Calculus Manifolds, Publisher University and vocational education Hanoi, 1985.

13. Burns Z. and Gidea M. - Differential Geometry and Topology With a View to Dynamical Systems, Chapman and Hall/CRC, 2005.

14. Wright Z. A. and Dixon K. - The Poincare-Hopf Theorem, The Open Mathematics Journal, 2008.

15. Guillemin Z. and Pollack A. - Differential Topology. Prentice-Hall, 1974.

16. Bott Z. and Tu L. - Differential Forms in Algebraic Topology, Springer, New York, 1982.

17. George C. - The RAISE Specification Language. Prentice Hall, NewYork, 1992.

\title{
TÓM TĂT
}

\section{KĨ THUẬT MÔ PHỎNG TÓC VÀ XOÁY TÓC DỰA VÀO TRƯỜNG VÉC TƠ TRÊN ĐA TAP}

\author{
Nguyễn Văn Huân \\ Truờng Đại học Công nghệ thông tin và Truyền thông - Đại học Thái Nguyên, Xã Quyết Thắng, \\ Thành phố Thái Nguyên, tỉnh Thái Nguyên \\ Email:nvhuan@ictu.edu.vn
}

Tóc là một thành phần quan trọng trong hệ thống mô phỏng con người ảo. Trong quá trình mô phỏng tóc, không những yêu cầu đảm bảo thời gian mà còn đòi hỏi phải biểu diễn được những đặc trưng vật lí, hoá học của tóc như kiểu dáng, màu sắc, độ cong, xoắn, tóc rẽ ngôi và kể cả xoáy tóc,,.. nhẳm thể hiện được tính chân thực và nâng cao chất lượng mô phỏng tóc. Trong khi, những công trình nghiên cứu đã công bố ngày nay mới tập trung vào mô phỏng được những kiểu dáng, chuyển động của tóc mà chưa biểu diễn được hết những đặc trưng của tóc như xoáy tóc, ...

Bài báo này đưa ra khái niệm da đầu như một đa tạp trong không gian 3 chiều. Dựa vào tính chất về điểm kì dị của trường vectơ trên đa tạp, bài báo đề xuất một kĩ thuật mô phỏng tóc trên bề mặt da đầu dựa vào trường vectơ trên đa tạp. Nhờ đó, có thể mô phỏng được xoáy tóc một cách phù hợp.

Tù khóa: da đầu, đa tạp, trường véc tơ, điểm kì dị, tóc. 